\title{
Study of Thyroid Lesions: Co-Relation of TIRADS with Bethesda System
} Dr. Bhavana Grandhi ${ }^{1 *}$, Dr. K. Durga ${ }^{2}$, Dr. N. Mohan Rao ${ }^{3}$, Dr. B. Syamasundara Rao ${ }^{4}$, Dr.M.Vijayalakshmi ${ }^{5}$, Dr. G.V. Sunandha Lakshmi ${ }^{6}$

\footnotetext{
${ }^{1}$ Associate Professor Department of Pathology Narayana Medical College Nellore-524003

${ }^{2}$ Professor \& HOD Department of Pathology Narayana Medical College Nellore-524003

${ }^{3,4}$ Professor Department of Pathology Narayana Medical College Nellore-524003

${ }^{5}$ Associate Professor Department of Pathology Narayana Medical College Nellore-524003

${ }^{6}$ Assistant Professor Department of Pathology Narayana Medical College Nellore-524003
}

DOI: $10.36348 /$ sjpm.2021.v06i04.002

| Received: 09.02.2021 | Accepted: 31.03.2021 | Published: 08.04.2021

*Corresponding author: Dr. Bhavana Grandhi

\section{Abstract}

Thyroid lesions are a common encounter in clinical practice. Standardisation in the reporting of these cases in radiology and cytology is a recent advance. TIRADS system is the standard in the reporting of radiology and Bethesda system (TBSRTC) has been used as the benchmark in cytology. We have undertaken this study with the aim of studying the patterns of thyroid lesions in cytology and compare the same with reporting system in radiology (TIRADS). All the cases coming to the Departments of Endocrinology \& Surgery were included and we have 47 cases with a female predominance, 43.7 years was was the mean age of presentation with female predominance, only 2 cases were seen in males. Benign lesions (80\%) was predominant over the malignant cases (10\%). Most of the cases come under category II in the TBSRTC system in cytology which includes nodular colloid goiter, adenomatous goiter $\&$ thyroiditis. Taking the TIRADS system into consideration, most of the cases were included under category 3(probably benign) with the malignancy rate as low as 5\%.Maximum concordance was seen between TIRADS 3 and TBSRTC IIresults. Thus our results were in par with other similar studies. Fine needle aspiration can thus be performed only in cases which belong to TIRADS IV or V and thus avoid unnecesary aspirations.

Keywords: Bethesda, TIRADS, Thyroid.

Copyright ( 12021 The Author(s): This is an open-access article distributed under the terms of the Creative Commons Attribution 4.0 International License (CC BY-NC 4.0) which permits unrestricted use, distribution, and reproduction in any medium for non-commercial use provided the original author and source are credited.

\section{INTRODUCTION}

Thyroid nodules are very common in clinical practice and the incidence ranges from $4-7 \%$ on palpation whereas on ultrasonographic examination the prevalence ranges from 50-60\% [1]. In the literature thyroid nodule can be defined as a discrete lesion in the thyroid gland which is radiologically distinct from the surrounding thyroid parenchyma, it can be solitary, multiple, solid or cystic and may or may not be functional. Thus ultrasound has considerably increased the number of cases identified, but has low accuracy in differentiating benign from malignant lesions [2]. Major concern regarding these nodules is to rule out or confirm malignancy by USG and FNA and surgical removal of all thyroid lesions can be evitable with their combined use [3].

For the provision of uniform diagnostic terminology for all the pathologists, a standardized category based evaluation proposed was TBSRTC: The
Bethesda System for Reporting Thyroid Cytopathology. The main advantage of this system of reporting is that it gives an implied risk of malignancy and recommends clinical management according to the category. Thyroid USG was standardized by the scoring system: TIRADS, the Thyroid Imaging Reporting and Data System, first proposed by Horvath et al. [4, 5]. This system has been established to standardize the scoring system along with recommendations for using FNA so that appropriate patient management can be done.

The present study makes an attempt to observe the concordance between these two systems on the thyroid lesions in the patients who have come to our hospital.

\section{MATERIALS \& METHODS}

We have done a prospective study from Janaury 2019 to January 2020 in the Department of Pathology. All the patients with lesions in the thyroid 
who have come to the FNAC section were evaluated. We have included the patients who were evaluated in the Department of Radiology with TIRADS scoring. Thus taking these criteria into consideration, we have included 47 patients in our study. The TIRADS scoring was given by a single radiologist.

\section{INCLUSION CRITERIA}

1. All the patients who came for fine needle aspiration with thyroid lesions that were given radiology scoring by TIRADS system.

2. Patients with thyroid lesions who were not diagnosed previously by TIRADS \& TBSRTC

\section{EXCLUSION CRITERIA}

1. Patients with thyroid lesions who were not given radiology scoring by TIRADS system.

2. Cases previously diagnosed as malignancy

These patients underwent fine needle aspiration cytology and the slides were reviewed and reported by a single pathologist using TBSRTC scoring. Statistical analysis was done.

\section{RESULTS}

In our study a total of 47 patients were included, out of which $45(95.44 \%)$ were females and only $2(4.25 \%)$ were males. The male to female ratio was $0.044: 1$. The female predominance may be related to autoimmunity which is very common in women along with hormonal imbalances. Age of the patients ranged from second decade to eighth decade, youngest being 21 year female and the oldest was 82 year old male. The mean age incidence was 43 .8years. The most common presenting complaint was asymptomatic swelling in front of neck. Ultrasound evaluation was done in all the cases. TIRADS scoring was given for all the cases by a single radiologist to avoid interobserver variability. Table 1 . Fine needle aspiration was done in all 47 cases and the smears were examined by a single pathologist and Bethesda scoring was given Table 2.

Table-1: TIRADS scoring of the various thyroid lesions

\begin{tabular}{|l|l|l|}
\hline Score & No of cases & \% of cases \\
\hline 1 & 1 & 2.13 \\
\hline 2 & 7 & 14.9 \\
\hline 3 & 28 & 59.57 \\
\hline 4 & 10 & 21.27 \\
\hline 5 & 1 & 2.13 \\
\hline 6 & 0 & - \\
\hline Total & 47 & 100 \\
\hline
\end{tabular}

Majority of the cases belonged to TIRADS category 3(probably benign), accounting for 59.57 of all the cases.

Table-2: TBSRTC scoring of the various thyroid lesions

\begin{tabular}{|l|l|l|}
\hline Score & No of cases & \% of cases \\
\hline I & 0 & - \\
\hline II & 38 & 80.86 \\
\hline III & 0 & - \\
\hline IV & 2 & 4.25 \\
\hline V & 2 & 4.25 \\
\hline VI & 5 & 10.64 \\
\hline Total & 47 & 100 \\
\hline
\end{tabular}

Majority of the cases belonged to TBSRTC Category II (benign), accounting for of all the cases.

Out of 38 cases in Bethesda Category II, majority of the cases $(60.5 \%)$ were in TIRADS 3.One case of discrepancy was seen where TIRADS was given as 6(malignancy). Most of the cases $(60 \%)$ in Bethesda category 6 were given as 4 and 1 cases was given as 6 in TIRADS scoring. Maximum concordance was seen between TBSRTC II and TIRADS 3.Table 3.

Table-3: Comparison of both the scoring systems

\begin{tabular}{|c|c|c|c|}
\hline TBSRTC & No of cases & TIRADS & No of cases \\
\hline I & 0 & & 0 \\
\hline II & 38 & $\begin{array}{l}1 \\
2 \\
3 \\
4 \\
6\end{array}$ & \\
\hline III & 0 & - & \\
\hline IV & 2 & 3 & \\
\hline $\mathrm{V}$ & 2 & $\begin{array}{l}3 \\
4\end{array}$ & \\
\hline VI & 5 & $\begin{array}{l}3 \\
4 \\
5\end{array}$ & \\
\hline Total & 47 & Total & 47 \\
\hline
\end{tabular}




\section{DISCUSSION}

The present study was undertaken to analyze the concordance between cytological evaluation (TBSRTC) and USG evaluation (TIRADS) on thyroid lesions. Fine needle aspiration of cytology is a cost effective tool with precise accuracy and can defer surgical intervention. In ideal conditions, clinical evaluation of thyroid lesions with thyroid function tests is followed by USG evaluation. FNA should then be performed on suspicious lesions

Most of the cases were in females in our study. The predominance of female population presenting with thyroid lesions is seen in many other studies [6-8]. This may be related to hormonal influences of estrogen and progesterone. Exposure to ionizing radiation or occupational exposures increases the incidence of nodules in thyroid. The mean age of the patients in our study, 43.8 years was in concordance with the studies done by Regmi et al. [9]

Regarding the laterality of the lesion, right lobe of the thyroid is most commonly affected in our study $(48.9 \%)$ and most of the lesions are nodular on inspection. Regmi et al. documented similar findings in her study.

Thyroid nodules are described according to the following categories - internal components, echogenecity, margins, calcifications \& shape. Internal components can be solid or cystic. Echogenecity may vary from hyperechogenic (isogenic) to hypogenic. Margins may be circumscribed or non-circumscribed. There may be microcalcifications or macrocalcifications including egg shell calcifications. Shape is described as parallel or non parallel. On ultrasound, the features which define malignancy are marked hypoechogenecity, non-circumscribed margins, microcalcifications or mixed calcifications and nonparallel shape [10]. The Thyroid Imaging Reporting and Data System (TIRADS) were proposed by Horvath et al. [5] to standardize the scoring system of thyroid USG. The categories under TIRADS are

Score 1: Normal Thyroid gland

Score 2: Benign conditions (0\% malignancy)

Score 3: Probably benign conditions (5\% malignancy)

Score 4: Suspicious nodules (5\% to $80 \%$ malignancy)

$4 \mathrm{a}$ - one feature of malignancy present

$4 \mathrm{~b}$ - two features of malignancy present

$4 c-$ three features of malignancy present

Score 5: Probably malignant (> 80\% malignancy)

Score 6: Biopsy proven malignant nodules

Regarding the standardisation in cytology reporting, The Bethesda System for Reporting Thyroid Cytopathology (TBSRTC) is being used. Six categories I to VI have been used in this system [11].
Category I: Non-diagnostic/material extracted is unsatisfactory for examination.

Category II: Benign-Benign follicular nodule

Hashimoto's thyroiditis/Lymphocytic

Granulomatous (sub-acute) thyroiditis

Category III: Atypia of undetermined significance

Follicular lesion of undetermined significance

Category IV: Follicular lesion

Suspicious of follicular neoplasm

Category V: Suspicious of malignancy

Category VI: Malignant lesion

In our study none of the lesions belonged to TBSRTC category I which includes non-diagnostic or satisfactory. The most common cause is the cystic nature of the lesion. Usually $1.8 \%$ to $23.6 \%$ cases belong to TBSRTC I and the percentage will be $7 \%$ even after repeat FNA procedure. So TBSRTC recommend that FNA smears sould have atlaest six groups of well preserved, well stained amd well visualized thyroid follicular epithelial cells and each group should be composed of ten(10)cells, preferably on a single slide $[11,12]$.

In our study $8.5 \%$ of the cases were malignant on FNA and if cases in TIRADS $\mathrm{V} \&$ TBSRTC $\mathrm{V}$ were included the prevalence rate of malignancy was $9.8 \%$. In the study done by Regmi et al. [9], it was $9.25 \%$ and $15.68 \%$ in the studies done by Bista M et al. [13] (study done in Nepal) Similar to our study, even in the study done by Vargas-Uricoechea $\mathrm{H}$ et al., ood concordance between TIRADS and TBSRTC [14].Thus, we can conclude that there is an increasing trend of malignancy of thyroid lesions all over the world which can be attributed to the availability of diagnostic facilities.

Different diagnostic categories have been proposed by TBSRTC for thyroid lesions on FNA to establish uniformity in reporting. TIRADS endorsed by the ACR recommends FNA to be performed in lesions which are suspicious for malignancy, TIRADS III, IV $\& \mathrm{~V}$.

\section{CONCLUSION}

In our study we have observed a good co relation between ultra sound guided diagnosis made by TIRADS and fine needle aspiration diagnosis by TBSRTC. By stratifying the thyroid lesions according to TIRADS, FNA can be performed only on suspicious lesions which can avoid unnecessary aspiration on thyroid lesions 


\section{ACKNOWLEDGEMENTS}

I thank my postgraduates, P. Dinusha, P.Viswanath and K. Samanth who have helped me in documenting the cases.

\section{REFERENCES}

1. Gharib, H., Papini, E., Garber, J. R., Duick, D. S., Harrell, R. M., Hegedus, L., ... \& Vitti, P. (2016). American Association of Clinical Endocrinologists, American College of Endocrinology, and Associazione Medici Endocrinologi Medical Guidelines for Clinical Practice for the Diagnosis and Management of Thyroid Nodules-2016 Update Appendix. Endocrine practice, 22, 1-60.

2. Gharib, H., Papini, E., Paschke, R., Duick, D. S., Valcavi, R., Hegedüs, L., \& Vitti, P. (2010). American Association of Clinical Endocrinologists, Associazione Medici Endocrinologi, and European Thyroid Association medical guidelines for clinical practice for the diagnosis and management of thyroid nodules: executive summary of recommendations. Journal of endocrinological investigation, 33(5), 287-291.

3. Tessler, F. N., Middleton, W. D., Grant, E. G., Hoang, J. K., Berland, L. L., Teefey, S. A., ... \& Stavros, A. T. (2017). ACR thyroid imaging, reporting and data system (TI-RADS): white paper of the ACR TI-RADS committee. Journal of the American college of radiology, 14(5), 587-595.

4. Cibas, E. S., \& Ali, S. Z. (2017). The 2017 Bethesda system for reporting thyroid cytopathology. Thyroid, 27(11), 1341-1346.

5. Horvath, E., Majlis, S., Rossi, R., Franco, C., Niedmann, J. P., Castro, A., \& Dominguez, M. (2009). An ultrasonogram reporting system for thyroid nodules stratifying cancer risk for clinical management. The Journal of Clinical Endocrinology \& Metabolism, 94(5), 1748-1751.

6. Vargas-Uricoechea, H., Meza-Cabrera, I., \& Herrera-Chaparro, J. (2017). Concordance between the TIRADS ultrasound criteria and the BETHESDA cytology criteria on the nontoxic thyroid nodule. Thyroid research, 10(1), 1-9.
7. Hong, M. J., Na, D. G., Baek, J. H., Sung, J. Y., \& Kim, J. H. (2017). Cytology-ultrasonography riskstratification scoring system based on fine-needle aspiration cytology and the Korean-thyroid imaging reporting and data system. Thyroid, 27(7), 953-959.

8. Periakaruppan, G., Seshadri, K. G., Krishna, G. V., Mandava, R., Sai, V. P., \& Rajendiran, S. (2018). Correlation between ultrasound-based TIRADS and Bethesda system for reporting thyroidcytopathology: 2-year experience at a tertiary care center in India. Indian journal of endocrinology and metabolism, 22(5), 651.

9. Regmi, S., Tiwari, A., \& Sharma, R. (2018). Comparison of Fine Needle Aspiration Cytology in Thyroid Lesions using The Bethesda System for Reporting Thyroid Cytopathology with Ultrasonography using Thyroid Imaging Reporting and Data System. Journal of Lumbini Medical College, 6(2).

10. Kim, E. K., Park, C. S., Chung, W. Y., Oh, K. K., Kim, D. I., Lee, J. T., \& Yoo, H. S. (2002). New sonographic criteria for recommending fine-needle aspiration biopsy of nonpalpable solid nodules of the thyroid. American Journal of Roentgenology, 178(3), 687-691.

11. Cibas, E.S., Ali, S.Z. (2009). The Bethesda System for Reporting Thyroid Cytopathology. Thyroid, 19(11):1159-1165

12. Ha, S. M., Ahn, H. S., Baek, J. H., Ahn, H. Y., Chung, Y. J., Cho, B. Y., \& Park, S. B. (2017). Validation of three scoring risk-stratification models for thyroid nodules. Thyroid, 27(12), 15501557.

13. Bista, M., Toran, K.C., Regmi, D., Maharaj, M., Kaple, P., Shrestha, S. (2011). Diagnostic accuracy of fine needle aspiration cytology in thyroid lesions: Journal of Nepal Health Res Counc, 9(18):14-6

14. Vargas-Uricoechea, H., Meza-Cabrera, I., \& Herrera-Chaparro, J. (2017). Concordance between the TIRADS ultrasound criteria and the BETHESDA cytology criteria on the nontoxic thyroid nodule. Thyroid research, 10(1), 1-9. 